\title{
Heavy-Hole States in Germanium Hut Wires
}

Hannes Watzinger, ${ }^{*}{ }^{\dagger, \ddagger}$ Christoph Kloeffel, ${ }^{*}, \S$ Lada Vukušić, ${ }^{\dagger \dagger}$ Marta D. Rossell, ${ }^{\|, \perp}$ Violetta Sessi, ${ }^{\#}$ Josip Kukučka, ${ }^{\dagger \neq}$ Raimund Kirchschlager, ${ }^{\dagger, \ddagger}$ Elisabeth Lausecker, ${ }^{\dagger,}$ Alisha Truhlar, ${ }^{\dagger, \ddagger}$ Martin Glaser, ${ }^{\dagger}$ Armando Rastelli, ${ }^{\ddagger}$ Andreas Fuhrer, ${ }^{\perp}$ Daniel Loss, ${ }^{\S}$ and Georgios Katsaros ${ }^{\dagger,+}$

${ }^{\dagger}$ Institute of Science and Technology Austria, Am Campus 1, 3400 Klosterneuburg, Austria

${ }^{\ddagger}$ Institute of Semiconductor and Solid State Physics, Johannes Kepler University, Altenbergerstrasse 69, 4040 Linz, Austria

${ }^{\S}$ Department of Physics, University of Basel, Klingelbergstrasse 82, 4056 Basel, Switzerland

"Electron Microscopy Center, Empa, Swiss Federal Laboratories for Materials Science and Technology, Überlandstrasse 129,8600 Dübendorf, Switzerland

${ }^{\perp}$ IBM Research Zürich, CH-8803 Rüschlikon, Switzerland

${ }^{\#}$ Chair for Nanoelectronic Materials, Technical University Dresden, 01062 Dresden, Germany

Supporting Information

ABSTRACT: Hole spins have gained considerable interest in the past few years due to their potential for fast electrically controlled qubits. Here, we study holes confined in Ge hut wires, a so-far unexplored type of nanostructure. Low-temperature magnetotransport measurements reveal a large anisotropy between the in-plane and out-of-plane gfactors of up to 18. Numerical simulations verify that this large anisotropy originates from a confined wave function of heavy-hole character. A light-hole admixture of less than $1 \%$ is estimated for the states of lowest energy, leading to a surprisingly large reduction of the out-of-plane g-factors compared with those for pure heavy holes. Given this tiny light-hole contribution, the spin lifetimes are expected to be very long, even in isotopically nonpurified samples.

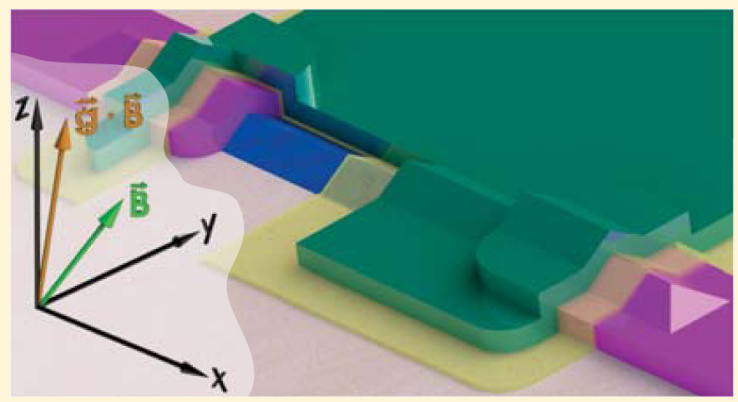

KEYWORDS: Germanium, quantum dot, heavy hole, g-factor, Luttinger-Kohn Hamiltonian

$\mathrm{T}$ he interest in group IV materials for spin qubits has been continuously increasing over the past few years after the demonstration of long electron spin decay times. ${ }^{1-5}$ Silicon $(\mathrm{Si})$ not only has the advantage of being the most important element in semiconductor industry; it can also be isotopically purified, eliminating the problem of decoherence from hyperfine interactions. Indeed, the use of such isotopically purified samples allowed the observation of electron spin coherence times of almost $1 \mathrm{~s} .{ }^{6}$ One limitation of $\mathrm{Si}$ is the difficulty in performing fast gate operations while maintaining good coherence. One way around this problem is to use the spin-orbit interaction of holes ${ }^{7}$ and manipulate the spin with electric fields. First steps in this direction have been recently reported. ${ }^{8}$ Holes in germanium (Ge) have an even stronger spin-orbit coupling. ${ }^{9-11}$ This fact together with the rather weak hyperfine interaction, already in nonpurified materials, make Ge quantum dots (QDs) a promising platform for the realization of high-fidelity spin qubits. $^{12}$

In 2002, the first $\mathrm{Ge}-\mathrm{Si}$ core-shell nanowires (NWs) were grown by chemical vapor deposition, ${ }^{13}$ and soon after, QDs were investigated in such structures. ${ }^{14-16}$ The cylindrical geometry of the NWs, however, leads to a mixture of heavy holes $(\mathrm{HH})$ and light holes (LH). ${ }^{9,17-19}$ As a consequence, the hyperfine interaction is not of Ising type, which thus reduces the spin coherence times. ${ }^{20}$ Still, spin relaxation times of about $600 \mu \mathrm{s}^{21}$ and dephasing times of about $200 \mathrm{~ns}^{22}$ were reported. One way of creating Ge QDs with noncylindrical symmetry is by means of the so-called Stranski-Krastanow (SK) growth mode. ${ }^{23}$ In 2010, the first single-hole transistors based on such SK Ge dome-like nanostructures were realized. ${ }^{24}$ Electrically tunable g-factors were reported, ${ }^{25}$ and Rabi frequencies as high as $100 \mathrm{MHz}$ were predicted. ${ }^{26}$ However, due to their very small size, it is difficult to create double-QD structures, typically used in spin-manipulation experiments. ${ }^{27}$ A solution to this problem can come from a second type of SK Ge nanostructures, the hut clusters, which were observed for the first time in $1990 .^{28}$ Zhang et al. ${ }^{29}$ showed in 2012 that under appropriate conditions, the hut clusters can expand into Ge hut wires (HWs), with lengths exceeding $1 \mu \mathrm{m}$. Two years later, the growth of $\mathrm{SiGe} \mathrm{HWs}$ was also demonstrated. ${ }^{30}$ HWs have a triangular cross-section with a height of about $2 \mathrm{~nm}$ above the wetting layer (WL) and are fully strained. These structural properties should lead to a very large $\mathrm{HH}-\mathrm{LH}$ splitting, minimizing the mixing and, as a consequence, the non-Ising type coupling to the nuclear spins. Despite this

Received: July 1, 2016

Revised: September 12, 2016

Published: September 22, 2016 


\section{(a)}
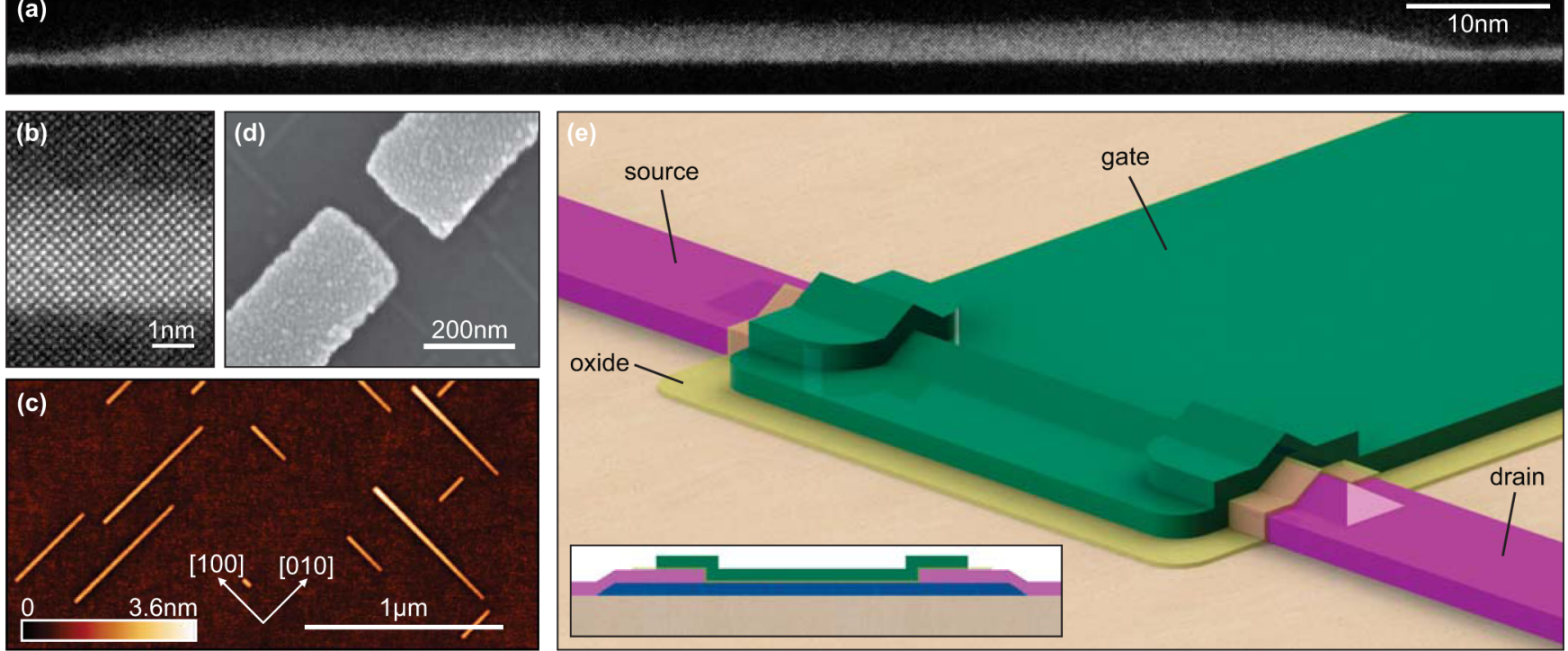

Figure 1. (a) Scanning transmission electron microscope image along a HW embedded in epitaxial Si. (b) Wire cross-section at higher resolution showing the defect-free growth of the wires. (c) Atomic force microscopy image of uncapped Ge HWs. (d) Scanning electron micrograph of a HW contacted by Pd source and drain electrodes. (e) Schematic representation of a processed three-terminal device studied in this work. The Ge HW, which is grown on a Si substrate, and its source and drain electrodes are covered by a thin hafnium oxide layer. The top gate covers the HW and partly the source and drain contacts. Correspondingly, the cross-section of the device along the HW (blue) is shown in the inset.

interesting perspective, not much is known about their electronic properties.

Here, we study three-terminal devices fabricated from Ge HWs. Scanning transmission electron microscopy (STEM) images verify that during their formation via annealing, no defects are induced. From magnetotransport measurements, a strong inplane vs out-of-plane g-factor anisotropy can be observed, and numerical simulations reveal that the low-energy states in the HWs are of $\mathrm{HH}$ type. The calculated results are consistent with the experimental data and confirm that confined holes in Ge are promising candidates for spin qubits.

The Ge HWs used in this study were grown by means of molecular beam epitaxy on 4-inch low-miscut $\mathrm{Si}(001)$ wafers, as described in ref 30 . Ge $(6.6 \AA)$ was deposited on a Si buffer layer, leading to the formation of hut clusters. After a subsequent annealing process of roughly $3 \mathrm{~h}$, in-plane Ge HWs with lengths of up to $1 \mu \mathrm{m}$ were achieved. In the last step of the growth process, the wires were covered with a $5 \mathrm{~nm}$ thick $\mathrm{Si}$ cap to prevent the oxidation of $\mathrm{Ge}$. The deposited $\mathrm{Si}$ acts also as a potential barrier that enables hole confinement. Figure 1a shows a STEM image taken with an annular dark-field detector. The Ge HW and the WL (bright) are surrounded by the Si substrate below and the Si cap on top (dark). The STEM lamella containing the HW was prepared along the [100] direction by focused ion beam milling and thinned to a final thickness of about $60 \mathrm{~nm}$. The STEM images show no signs of dislocations or defects, indicating perfect heteroepitaxy (see also Figure $1 \mathrm{~b}$ ). The height of the encapsulated wires is about 20 monolayers $(2.8$ $\mathrm{nm}$ ), including the WL. Besides having well-defined triangular cross-sections, the HWs are oriented solely along the [100] and the [010] directions, as can be seen in the atomic force micrograph of uncapped Ge HWs in Figure 1c.

For the fabrication of three-terminal devices, metal electrodes were defined by electron beam lithography. After a short oxide removal step with buffered hydrofluoric acid, $30 \mathrm{~nm}$ thick palladium (Pd) contacts were evaporated. The gap between source and drain electrodes ranges from 70 to $100 \mathrm{~nm}$ and is illustrated in Figure 1d. The sample was then covered by a $10 \mathrm{~nm}$ thick hafnium oxide insulating layer. As a last step, top gates consisting of $\mathrm{Ti} / \mathrm{Pd}(3 / 20 \mathrm{~nm})$ were fabricated. A schematic representation of a processed $\mathrm{HW}$ device is depicted in Figure 1e.

The devices were cooled in a liquid $\mathrm{He}-3$ refrigerator with a base temperature of about $250 \mathrm{mK}$ equipped with a vector magnet. The sample characterization was performed using lownoise electronics and standard lock-in techniques.

In the following, the results of two similar devices are presented that only differ slightly in the gap size between source and drain; the two devices have channel lengths of 95 and $70 \mathrm{~nm}$, respectively. A stability diagram of the first device is shown in Figure 2a. Closing Coulomb diamonds prove that a single QD formed in the HW. Typical charging energies lie between 5 and $10 \mathrm{meV}$, and excited states can be clearly observed. The corresponding level spacing between the ground states and the first excited states is up to $1 \mathrm{meV}$. Because at more positive gate voltages, the current signal becomes too small to be measured, we cannot define the absolute number of holes confined in the QD. At least 10 more charge transitions after the $2 \mathrm{~N}$ state can be observed for more positive gate voltages. To get additional information, the device was cooled down a second time and measured at $4 \mathrm{~K}$ by radio frequency dispersive reflectometry. ${ }^{31}$ The reflectometry signal did not reveal the existence of additional holes beyond the regime in which the current signal vanished, which, however, could be also due to the slow tunnel rates. We can thus just estimate that in the discussed crossings, the number of holes is about 10 to 20 , i.e., the QD states most likely form from the first sub-band.

For holes, the band structure is more complex than for electrons. At the $\Gamma$ point, the $\mathrm{HH}$ and $\mathrm{LH}$ bands are degenerate. This degeneracy can be lifted by strain and confinement. ${ }^{32}$ In two-dimensional hole gases of semiconductors with compressive, biaxial in-plane strain, the $\mathrm{HH}$ states lie lower in energy than the LH states, i.e., it would cost less energy to excite an electron to the conduction band from the $\mathrm{HH}$ than the $\mathrm{LH}$ band. ${ }^{33}$ 


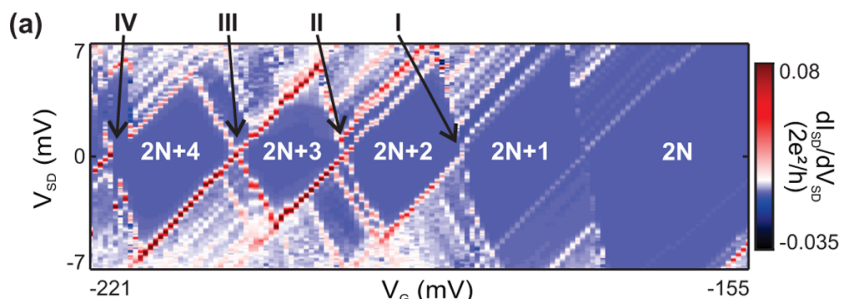

$\left(\mathrm{d} I_{\mathrm{SD}} / \mathrm{d} V_{\mathrm{SD}}\right)$ vs gate $\left(V_{\mathrm{G}}\right)$ and source-drain voltage $\left(V_{\mathrm{SD}}\right)$ at various magnetic fields are presented. The signature of a singly occupied doubly degenerate level is the appearance of an additional line ending at both sides of the diamond once a magnetic field is applied. These extra lines are indicated by black arrows in panels $\mathrm{c}$ and $\mathrm{d}$ of Figure 2 for crossing IV, in panels $\mathrm{g}$ and $\mathrm{h}$ for crossing III, and in panel $\mathrm{m}$ for crossing I. They allow us to identify the diamonds between crossing II and III and on the right side of crossing I as diamonds with an odd number of confined holes.

In addition, from the position of these extra lines, the Zeeman energy $E_{Z}=g \mu_{B} B$ can be extracted with $\mu_{B}$ the Bohr magneton, $B$ $=|B|$, and $g$ standing for the absolute value of the g-factor. By the plotting of the Zeeman energies vs the magnetic field and the application of a linear fit to the data, forced through the origin, the hole g-factor can be determined (see Figure 2o). For crossing IV and an out-of-plane magnetic field $B_{z}$ we determine that $g_{\perp}=$ $3.07 \pm 0.31$. The same type of measurements result in a slightly higher value of the $g_{\perp}$-factor for the diamonds with a smaller amount of holes. Compared to the out-of-plane magnetic field, the in-plane directions have an almost negligible effect on the hole state splitting, as shown in Figure 2e for crossing IV and in (i) for crossing III, both at $B_{x}=3 \mathrm{~T}$. Due to the thermal broadening, the split lines can be barely resolved. Therefore, an upper limit of the g-factor is given for these cases. The lower parts of crossings II and I at $B_{y}=9 \mathrm{~T}$ are shown in panels $\mathrm{k}$ and $\mathrm{m}$ of Figure 2, respectively, where only the latter shows an observable splitting. The small g-factors for both in-plane magnetic fields lead to large g-factor anisotropies $z / x$ and $z / y$, ranging from 5 to about 20, as shown in the table in Figure 2p. A similar anisotropy was observed in crossing IV (III) for the triplet-like splitting indicated by white arrows in Figure $2 \mathrm{c}$ and $\mathrm{d}$ ( $\mathrm{g}$ and $\mathrm{h}$ ), resulting in $g_{\perp}=2.61 \pm 0.56$; the corresponding in-plane splitting is too small to be resolved at $250 \mathrm{mK}$. When the measured g-factors are compared with those reported for dome-like Ge QDs, ${ }^{24,26}$ it is observed that HWs have larger $g_{\perp}$ and much larger anisotropies, which are both characteristics of $\mathrm{HH}$ states. ${ }^{33,34}$

To validate whether our findings are general characteristics of

Figure 2. (a) Stability diagram of a HW device taken at $\sim 250 \mathrm{mK}$ and zero magnetic field. The number of confined holes is indicated in white and the relevant crossings are labeled with roman numerals. The shown color scale is also valid for panels $\mathrm{b}-\mathrm{m}$. $(\mathrm{b}-\mathrm{e})$ Differential conductance measurements vs $V_{\mathrm{G}}(x$-axis $)$ and $V_{\mathrm{SD}}\left(y\right.$-axis) for crossing IV for $B_{z}=0$, 1 , and 2 Tesla $(\mathrm{T})$ and $B_{x}=3 \mathrm{~T}$, respectively. Similarly, panels $\mathrm{f}-\mathrm{h}$ show the differential conductance of the lower half of crossing III vs $V_{\mathrm{G}}$ and $V_{\mathrm{SD}}$ for $B_{z}=0,1$, and $2 \mathrm{~T}$ and (i) for $B_{x}=3 \mathrm{~T}$. Measurements of crossing II are shown in panel $\mathrm{j}$ for $0 \mathrm{~T}$ and in panel $\mathrm{k}$ for $B_{y}=9 \mathrm{~T}$. Likewise, panels 1 and $\mathrm{m}$ show the lower part of crossing $\mathrm{I}$ at $0 \mathrm{~T}$ and $B_{y}=9 \mathrm{~T}$, respectively. For all measurements shown in panels $b-m$, the gate range is roughly $6 \mathrm{mV}$. In panel $\mathrm{n}$, the used nomenclature for the magnetic field orientations is illustrated. (o) Dependence of the Zeeman energy $E_{\mathrm{Z}}$ of the ground state in crossing IV vs $B_{z}$. The g-factors are extracted from the linear fit (red line). The measured g-factors for the three different magnetic field orientations, as well as the resulting anisotropies $z / x$ and $z / y$, are listed in (p) for crossings I to IV.

However, further carrier confinement can induce a strong mixture of $\mathrm{HH}$ and $\mathrm{LH}$ states. ${ }^{34}$

To investigate the nature of the HW hole states, their g-factors were determined via magnetotransport measurements. In the presence of an external magnetic field $\boldsymbol{B}$, the doubly degenerate QD energy levels split. For more than 15 diamond crossings, the Zeeman splitting was measured for the three orientations illustrated in Figure 2n. In Figure 2a-m, measurements of four representative crossings showing the differential conductance

HW devices, a second device was also measured. Figure 3 a shows the overview stability diagram with a focus on crossing $i$ and ii. Due to reasons of visibility, the corresponding magnetic field spectroscopy measurements are partly shown in current representation. In Figure $3 b-e$ and $f-i$, the dependence on the three different B-field orientations is illustrated for crossing ii and i, respectively. Inelastic cotunneling measurements for $2 \mathrm{~N}+5$ holes are shown in Figure $3 \mathrm{j}-1$ as a function of $B_{x}, B_{y}$, and $B_{z}$, respectively. The obtained g-factors are listed in the table in Figure $3 \mathrm{~m}$, with the highest out-of-plane g-factor being 4.3, similar to the first device. For the in-plane g-factors, $g_{x}$ or $g_{y}$, slightly increased values can be observed (because $g_{x}$ and $g_{y}$ show comparable values, they will be referred to as $g_{\|}$in the following). However, the g-factors in out-of-plane direction are still 10 times larger than for the in-plane orientation.

From the listed $\mathrm{g}$-factor values, two interesting observations can be made. First, as for the first device, the $g_{\perp}$-factor is decreasing for a higher number of holes, and second, the $g_{\|}$factors have clearly increased for a larger number of holes. As a consequence, a decrease of the anisotropies to less than 3 was observed for the $2 \mathrm{~N}+5$ hole state, indicating an increased $\mathrm{LH}$ contribution. $^{33,34}$

To get a better understanding of the measured g-factor values and their anisotropies, we consider a simple model for hole states in HWs. Taking into account the $\mathrm{HH}$ and $\mathrm{LH}$ bands of $\mathrm{Ge}$ and 
(a)
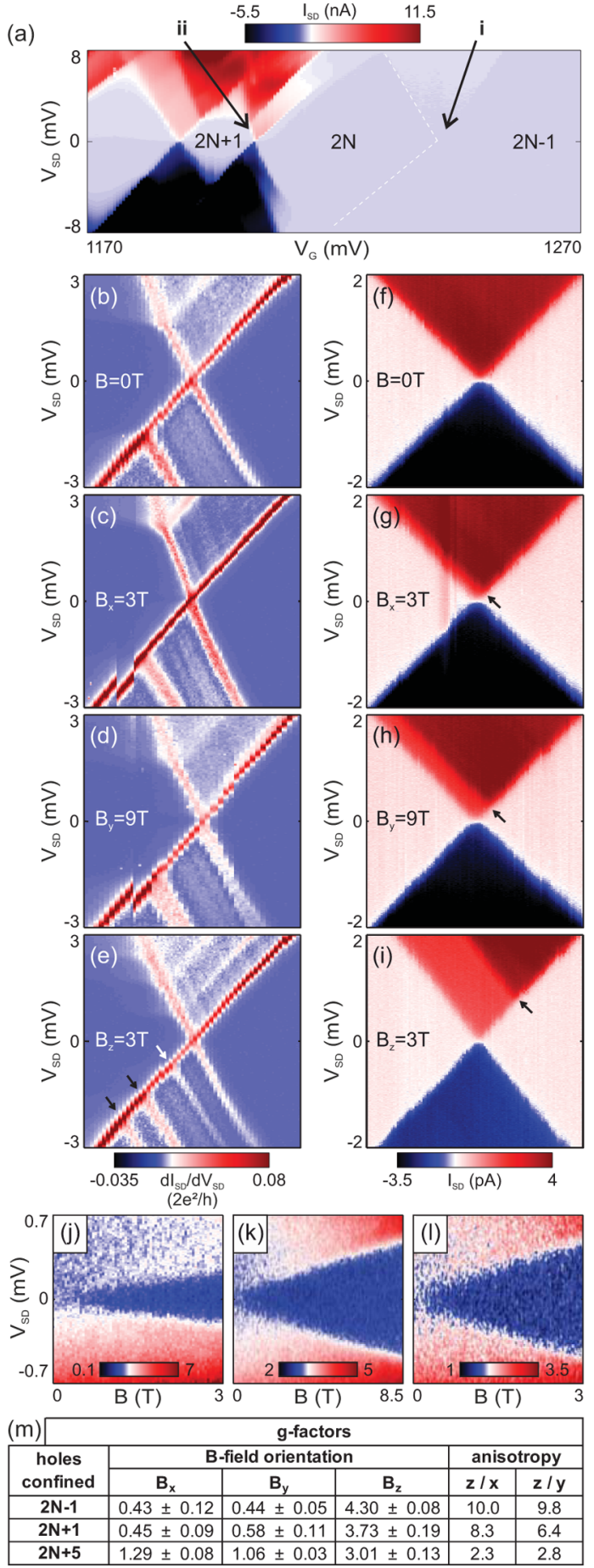

Figure 3. (a) Stability diagram of the second device with a focus on the crossings denoted as $i$ and ii. The magnetic field dependence is shown in panels $\mathrm{b}-\mathrm{e}$ for crossing $\mathrm{ii}$ and in panels $\mathrm{f}-\mathrm{i}$ for crossing i. For crossing ii, the splitting of the excited state can also be observed, as indicated in panel e by black arrows. The corresponding g-factors were extracted as $g_{\perp}=3.79 \pm 0.45, g_{x}<1.30, g_{y}<0.68$. Panels $j-1$ show differential conductance plots of inelastic cotunneling measurements for the $2 \mathrm{~N}+5$ hole state vs $V_{\mathrm{SD}}$ and $\mathrm{B}$ for $B_{x}, B_{y}$, and $B_{z}$, from left to right, respectively. The color scale insets indicate the differential conductance in units of $2 e^{2} / \mathrm{h} \cdot 10^{-4}$. In panel $\mathrm{m}$, the determined g-factor values and the corresponding anisotropy factors for the ground state of the discussed crossings are listed. The g-factors were determined from direct tunneling except the values for $2 \mathrm{~N}+1$ holes at $B_{x}=3 \mathrm{~T}$ and for $2 \mathrm{~N}+5$ holes, which were obtained from inelastic cotunneling measurements. assuming that the HWs are free of shear strain, our model Hamiltonian in the presence of a magnetic field is

$$
\begin{aligned}
H= & \frac{\hbar^{2}}{2 m}\left[\left(\gamma_{1}+\frac{5 \gamma_{2}}{2}\right) k^{2}-2 \gamma_{2} \sum_{\nu} k_{\nu}^{2} J_{\nu}^{2}\right. \\
& \left.-4 \gamma_{3}\left(\left\{k_{x}, k_{y}\right\}\left\{J_{x}, J_{y}\right\}+c p\right)\right] \\
& +2 \mu_{B} \mathbf{B} \cdot(\kappa \mathbf{J}+q \mathcal{J})+b \sum_{\nu} \epsilon_{\nu \nu} J_{\nu}^{2}+V(y, z)
\end{aligned}
$$

It comprises the Luttinger-Kohn Hamiltonian, ${ }^{35}$ the Bir-Pikus Hamiltonian, ${ }^{36}$ and the confinement in the transverse directions $V(y, z)$, for which we take a rectangular hard-wall potential of width $L_{y}$ and height $L_{z}$ for simplicity, i.e., $V(y, z)=0$ if both $|y|<L_{y} /$ 2 and $|z|<L_{z} / 2$ and $V(y, z)=\infty$ otherwise. In eq 1 , we omitted the term $-(a+5 b / 4)\left(\epsilon_{x x}+\epsilon_{y y}+\epsilon_{z z}\right)$ from the Bir-Pikus Hamiltonian, where $a$ is the hydrostatic deformation potential. This term corresponds to a global energy shift in our model and therefore cannot affect the results. In an extended model, where the energy of the valence band edge matters, this term should be included. We note that $-H$ refers to the valence band electrons, and a global minus was applied for our description of holes (which are unfilled valence band states). In eq $1,\{A, B\}=(A B+$ $B A) / 2$, "cp" are cyclic permutations, $m$ is the bare electron mass, and $J_{\nu}$ are dimensionless spin-3/2 operators. The subscript $\nu$ stands for the three axes $x, y, z$, which are oriented along the length, width, and height, respectively, of the HW (see Figures $2 \mathrm{n}$ and $4 a$ ) and coincide with the main crystallographic axes. With the listed vector components referring to the unit vectors along these three directions, the magnetic field is $\boldsymbol{B}=\left(B_{x}, B_{y}, B_{z}\right)$ and, furthermore, $J=\left(J_{x}, J_{y}, J_{z}\right), \mathcal{J}=\left(J_{x}^{3}, J_{y}^{3}, J_{z}^{3}\right)$. The operators $k_{\nu}$ are components of the kinetic electron momentum $\hbar k=-i \hbar \nabla+e A$, where $e$ is the elementary positive charge, $\nabla$ is the Nabla operator, and $\boldsymbol{B}=\nabla \times \boldsymbol{A}$. For the vector potential, we choose a convenient gauge $\boldsymbol{A}=\left(B_{y} z-B_{z} y,-B_{x} z / 2, B_{x} y / 2\right)$, and we note that $k^{2}=k \cdot k$.

The Hamiltonian $H$ of eq 1 may be written in matrix form by projection onto a suitable set of basis states. In agreement with the boundary conditions, we use the basis states ${ }^{18}$

$$
\left|j_{z}, n_{z}, n_{y}, \tilde{k}_{x}\right\rangle=\left|j_{z}\right\rangle \otimes\left|\varphi_{n_{z}, n_{y}, \tilde{k}_{x}}\right\rangle
$$

with orbital part

$$
\begin{aligned}
\varphi_{n_{z}, n_{y}, \tilde{k}_{x}}(x, y, z)= & \frac{2}{\sqrt{L_{z} L_{y}}} \times \sin \left[n_{z} \pi\left(\frac{z}{L_{z}}+\frac{1}{2}\right)\right] \\
& \times \sin \left[n_{y} \pi\left(\frac{y}{L_{y}}+\frac{1}{2}\right)\right] e^{i \tilde{k}_{x} x}
\end{aligned}
$$

where the $n_{z} \geq 1$ and $n_{y} \geq 1$ are integer quantum numbers for the transverse sub-bands, and $\tilde{k}_{x}$ is a wavenumber. Eq 3 applies when both $|y|<L_{y} / 2$ and $|z|<L_{z} / 2$, otherwise $\varphi_{n_{z}, n_{y}, \tilde{k}_{x}}=0$. The spin states $\left|j_{z}\right\rangle$ are eigenstates of $J_{z}$ and satisfy $J_{z}\left|j_{z}\right\rangle=j_{z}\left|j_{z}\right\rangle$, where $j_{z} \in\{3 / 2$, $1 / 2,-1 / 2,-3 / 2\}$. Because of the very strong confinement along $z$ in our system, basis states with $j_{z}= \pm 3 / 2\left(j_{z}= \pm 1 / 2\right)$ correspond to $\mathrm{HH}(\mathrm{LH})$ states. To analyze the low-energy properties of $H$, we project it onto the 36 dimensional subspace with $n_{z} \leq 3$ and $n_{y} \leq 3$. This range of sub-bands is large enough to 
(a)

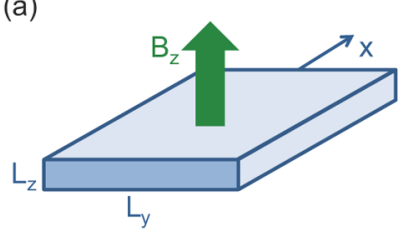

(c)
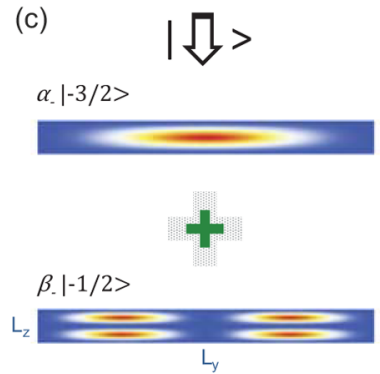

Figure 4. (a) Sketch of the HW model in the theoretical analysis. The cross-section is approximated by a rectangle of width $L_{y}$ and small thickness $L_{z}$. The green arrow represents an out-of-plane magnetic field $B_{z}$. (b) Effective four-level system used to derive the dominant correction $g_{\mathrm{C}}$ (eq 5) in the out-of-plane g-factor $g_{\perp} \simeq 6 \kappa+27 q / 2+g_{\mathrm{C}}$. The LH states $| \pm 1 / 2,2,2,0\rangle$ and the HH states $| \pm 3 / 2,1,1,0\rangle$ (see eqs 2 and 3 for details) differ by an energy of order $\Delta$. In the presence of $B_{z}$, the couplings between these states have the form $C_{ \pm}=C_{0} \pm \lambda B_{z}$. That is, zero-field couplings of equal strength $\left(C_{0}\right.$, gray dotted arrows) are enhanced and reduced, respectively $\left( \pm \lambda B_{z}\right.$ with a proportionality factor of $\lambda$, green dotted arrows), which results in $\left|C_{-}\right|<\left|C_{+}\right|$for $B_{z}>0$ as sketched in the diagram. (c) The Zeeman split eigenstates of lowest energy after diagonalization of the system in panel $b$. The ground state $\alpha_{-}|-3 / 2,1,1,0\rangle+\beta_{-}|-1 / 2,2,2,0\rangle$ (left, pseudospin down) consists of a $\mathrm{HH}$ state with spin $|-3 / 2\rangle$ with a probability density that has a peak at the center of the HW cross-section and a LH state with spin $|-1 / 2\rangle$ and four peaks near the corners (analogous for the excited state shown on the right, pseudospin up). The plots for the probability densities are dimensionless and correspond to $L_{z} L_{y}\left|\varphi_{1,1,0}\right|^{2}$ and $L_{z} L_{y}\left|\varphi_{2,2,0}\right|^{2}$, respectively (eq 3 ). We find $\left|\alpha_{ \pm}\right|^{2}>0.99$ for typical parameters, so the $\mathrm{LH}$ admixtures are very small. However, due to $\left|C_{-}\right|<\left|C_{+}\right|$caused by $B_{z}$ the LH admixtures $\left|\beta_{-}\right|^{2}<\left|\beta_{+}\right|^{2}$ differ slightly, as illustrated by the different plus signs (green) and the different LH contributions (black, not to scale) in the arrows for the pseudospin. This difference is associated with a substantial reduction of $g_{\perp}$; see $g_{C}$. The gray plus signs of equal size in the background refer to the initial couplings, which are reduced or enhanced, respectively, in the presence of $B_{z}$.

account for the most important couplings and small enough to enable fast numerical diagonalization.

The band structure parameters of (bulk) Ge are $\gamma_{1}=13.35, \gamma_{2}$ $=4.25, \gamma_{3}=5.69, \kappa=3.41$, and $q=0.07 ;^{37,38}$ the deformation potential is $b=-2.5 \mathrm{eV} .{ }^{36}$ The values for the strain tensor elements $\epsilon_{x x}=-0.033=\epsilon_{y y}$ and $\epsilon_{z z}=0.020$ are obtained from finite element simulations, as described in the Supporting Information. That is, the Ge lattice in the HW has almost completely adopted the lattice constant of Si along the $x$ and $y$ directions and experiences tensile strain along the out-of-plane direction $z$. Using moderate magnetic fields (of the order of Tesla) as in the experiment, $L_{y}=20 \mathrm{~nm}, L_{z} \leq 3 \mathrm{~nm}$, and the above-mentioned parameters, we diagonalize the resulting $36 \times$ 36 matrix numerically and find that the eigenstates of lowest energy are close-to-ideal $\mathrm{HH}$ states. They feature spin expectation values $\left\langle J_{z}\right\rangle$ above 1.49 and below -1.49 , respectively, when $\boldsymbol{B}$ is along $z$, and $\left\langle J_{\nu}\right\rangle \simeq 0$ for all $\nu \in\{x, y, z\}$ when $\boldsymbol{B}$ is in-plane. This corresponds to a $\mathrm{LH}$ admixture of less than $1 \%$. (An upper bound for the $\mathrm{LH}$ probability $p_{\mathrm{LH}}$ is given by $\left(1-p_{\mathrm{LH}}\right) 3 / 2+p_{\mathrm{LH}}$
$1 / 2>1.49$, which results in $p_{\mathrm{LH}}<0.01$.) Furthermore, the admixture remains very small even when electric fields that may have been present in the experiment are added to the theory (see the Supporting Information).

The numerically observed $\mathrm{HH}$ character of the low-energy states in our model can easily be understood. First, with $\epsilon_{x x}=\epsilon_{y y}$ $=\epsilon_{\|}$, the spin-dependent part of the strain-induced Hamiltonian can be written in the form $b\left(\epsilon_{z z}-\epsilon_{\|}\right) J_{z}^{2}$, and so basis states with $j_{z}$ $= \pm 1 / 2$ are shifted up in energy by more than $250 \mathrm{meV}$ compared to those with $j_{z}= \pm 3 / 2$. Second, the strong confinement along $z$ leads to an additional HH-LH splitting of the order of $\hbar^{2} \pi^{2}\left(m_{\mathrm{LH}}^{-1}-m_{\mathrm{HH}}^{-1}\right) /\left(2 L_{z}^{2}\right)$, where $m_{\mathrm{LH}}=m /\left(\gamma_{1}+2\right.$ $\left.\gamma_{2}\right)$ and $m_{\mathrm{HH}}=m /\left(\gamma_{1}-2 \gamma_{2}\right)$. This results in a large splitting of 2 $\gamma_{2} \hbar^{2} \pi^{2} /\left(m L_{z}^{2}\right) \geq 710 \mathrm{meV}$ for $L_{z} \leq 3 \mathrm{~nm}$.

The result that hole states with $j_{z}= \pm 1 / 2$ are so much higher in energy than those with $j_{z}= \pm 3 / 2$ suggests that one may simplify the Hamiltonian of eq 1 by projection onto the $\mathrm{HH}$ subspace, which is described in detail in the Supporting Information. If the LH states are ignored, one expects small in-plane g-factors $g_{\|} \simeq 3 q$ $\simeq 0.2$ and very large out-of-plane g-factors $g_{\perp} \simeq 6 \kappa+27 q / 2 \simeq$ 21.4 (see the Supporting Information). ${ }^{39}$ While $g_{\|}$is indeed small in our experiment and $g_{\perp} \gg g_{\|}$is indeed observed, the measured value of $g_{\perp}$ is significantly smaller than the one obtained from the pure-HH approximation.

When we diagonalize the $36 \times 36$ matrix, we find that the inplane g-factors are close to $3 q$, as also expected, e.g., from studies of the in-plane g-factors in narrow [001]-grown quantum wells. ${ }^{38,39,40}$ Our results for $g_{\|}$agree well with the experiment and are consistent with the $\mathrm{HH}$ character of the low-energy states. Rather surprisingly, however, even though the low-energy eigenstates consist almost exclusively of either $|3 / 2\rangle$ or $|-3 / 2\rangle$ when the magnetic field is applied along $z$, we also find that the resulting $g_{\perp} \approx 15$ is indeed smaller than the value expected from the pure-HH approximation. The reason is that, in fact, the tiny admixtures from the LH bands are not negligible for the g-factors, as illustrated in Figure 4 and described in the following. When the magnetic field is applied along the $z$ axis, the Zeeman split states of lowest energy consist mostly of $|-3 / 2,1,1,0\rangle$ and $\mid 3 / 2,1,1$, $0\rangle$, respectively. It turns out that the corresponding $g_{\perp}$ is strongly affected by the couplings

$$
C_{ \pm}=\langle \pm 3 / 2,1,1,0|H| \pm 1 / 2,2,2,0\rangle
$$

because they satisfy $\left|C_{+}\right| \neq\left|C_{-}\right|$in the presence of $B_{z}$ and therefore lead to different $\mathrm{LH}$ admixtures in the low-energy eigenstates of the HW (see the Supporting Information). The splitting between the basis states $| \pm 1 / 2,2,2,0\rangle$ and $I \pm 3 / 2,1,1,0\rangle$ in our model is predominantly determined by the confinement and can be approximated by $\Delta=\hbar^{2} \pi^{2}\left(4 m_{\mathrm{LH}}^{-1}-m_{\mathrm{HH}}^{-1}\right) /\left(2 L_{z}^{2}\right)$ using $L_{z} \ll L_{y}$. From second-order perturbation theory, ${ }^{38}$ we therefore find that the couplings of eq 4 lead to a correction

$$
g_{C}=\frac{\left|C_{-}\right|^{2}-\left|C_{+}\right|^{2}}{\mu_{B} B_{z} \Delta}=-\frac{2^{17} \gamma_{3}^{2}}{81 \pi^{4}\left(3 \gamma_{1}+10 \gamma_{2}\right)}
$$

to the out-of-plane g-factor $g_{\perp} \simeq 6 \kappa+27 q / 2+g_{C}$. Eq 5, whose derivation is explained in detail in the Supporting Information, contains the factor $2^{17} /\left(81 \pi^{4}\right) \simeq 16.6$ and, remarkably, depends solely on the three Luttinger parameters $\gamma_{1,2,3}$. With the parameters of $\mathrm{Ge}$, this formula yields $g_{C} \simeq-6.5$, which is a substantial reduction of $g_{\perp}$ due to orbital effects. ${ }^{18,41}$ Of course, $H$ couples $| \pm 3 / 2,1,1,0\rangle$ not only with $| \pm 1 / 2,2,2,0\rangle$ but also with other states. However, even when we take a large number of $10^{4}$ basis states into account $\left(n_{y}, n_{z} \leq 50\right)$ and calculate the 
admixtures to $| \pm 3 / 2,1,1,0\rangle$ via perturbation theory, we find that the sum of all corrections to $g_{\perp}$ is still close to $g_{C}$, i.e., eqs 4 and 5 describe the dominant part.

We note that if the $\mathrm{HH}-\mathrm{LH}$ splitting in our model were dominated by the strain, such that $\Delta$ in eq 5 were much greater than the splitting caused by the confinement, the correction to $g_{\perp}$ from LH states would be suppressed, and the model Hamiltonian would indeed approach the pure-HH approximation for the lowenergy states (see the Supporting Information). Moreover, we found in our calculations that magnetic-field-dependent corrections to the $\mathrm{g}$-factors are negligible given our $\mathrm{HW}$ parameters. This is consistent with $\sqrt{\hbar /(\mathrm{eB})}>L_{y} / 2$ for $B \leq$ $6.5 \mathrm{~T}$, where $\sqrt{\hbar /(\mathrm{eB})}$ is the magnetic length, and agrees well with the experiment (see, e.g., Figure 2o, where the measured Zeeman energy is proportional to the applied magnetic field).

Although the result $g_{\perp} \approx 15$ from our simple model is already smaller than $g_{\perp} \approx 21$ from the pure- $\mathrm{HH}$ approximation, it is still larger than the measured values. We believe that this remaining deviation is mainly due to the following three reasons. First, given the small height of the HW, the eigenenergies in our model approach or even exceed the valence band offset $\sim 0.5 \mathrm{eV}$ between $\mathrm{Ge}$ and $\mathrm{Si}^{15}$ and so the hole wave function will leak into the surrounding $\mathrm{Si}$. This certainly leads to a reduction of $g_{\perp}$ because the values of $\kappa$ in Ge and $\mathrm{Si}$ have opposite signs. ${ }^{37,38}$ Second, we used here the parameters of bulk Ge for simplicity. However, the strong confinement and the strain change the gaps between the various bands of the semiconductor, which (among other things) may lead to a substantial rescaling of the effective band structure parameters. ${ }^{38}$ Improvements can be expected from an extended model that also involves the split-off band and the conduction band. ${ }^{34,41,42}$ Finally, although our assumption of an infinite $\mathrm{HW}$ with a rectangular cross-section is a reasonable approximation for the elongated HW QDs realized here, the details of the confinement (and the strain) along all spatial directions can provide additional corrections. Taking all these elements fully into account is beyond the scope of the present work and requires extensive numerics.

In summary, having analyzed our HW model in detail, we can conclude that it reproduces all the key features of our experimental data and provides useful insight. It predicts a large g-factor anisotropy with $g_{\|}$close to zero and $g_{\|} \ll g_{\perp}<6 \kappa$, as seen in the experiment. The spin projections calculated with our model suggest that the low-energy states of HWs are almost pure $\mathrm{HHs}$ and that the tiny admixtures from energetically higher LH states lead to a substantial reduction of $g_{\perp}$, which is a consequence of the orbital part of the magnetic-field-coupling. Finally, keeping in mind the finite valence band offset between $\mathrm{Ge}$ and $\mathrm{Si}$, a possible explanation for the increasing $g_{\|}$and the decreasing $g_{\perp}$ observed experimentally with increasing occupation number is that the confinement caused by the $\mathrm{Ge}-\mathrm{Si}$ interface becomes less efficient as the eigenenergy of the hole increases (also due to the Coulomb repulsion, which leads to an additional charging energy if more than one hole is present). Hence, a larger occupation number may change the effective aspect ratios of the HW QD experienced by the added hole and, thus, increase its $\mathrm{HH}-\mathrm{LH}$ mixing.

\section{ASSOCIATED CONTENT}

\section{S Supporting Information}

The Supporting Information is available free of charge on the ACS Publications website at DOI: 10.1021/acs.nanolett.6b02715.
Additional details on finite element simulations of the strain distribution in a HW, matrix representation of the spin operators, calculation with electric fields, couplings, correction $g_{\mathrm{C}}$ to the out-of-plane g-factor, and Hamiltonian for pure heavy holes. A figure showing COMSOL simulations of the out-of-plane and the in-plane strain distribution in a capped HW. (PDF)

\section{AUTHOR INFORMATION}

\section{Corresponding Authors}

*E-mail: hannes.watzinger@ist.ac.at.

*E-mail: c.kloeffel@unibas.ch.

\section{Notes}

The authors declare no competing financial interest.

\section{ACKNOWLEDGMENTS}

The work was supported by the EC FP7 ICT project SiSPIN no. 323841, the EC FP7 ICT project PAMS no. 610446, the ERC Starting Grant no. 335497, the FWF-I-1190-N20 project, and the Swiss NSF. We acknowledge F. Schäffler for fruitful discussions related to the hut wire growth and for giving us access to the molecular beam epitaxy system, M. Schatzl for her support in electron beam lithography, and V. Jadriško for helping us with the COMSOL simulations. Finally, we thank G. Bauer for his continuous support.

\section{REFERENCES}

(1) Morello, A.; Pla, J. J.; Zwanenburg, F. A.; Chan, K. W.; Tan, K. Y.; Huebl, H.; Mottonen, M.; Nugroho, C. D.; Yang, C.; van Donkelaar, J. A.; Alves, A. D. C.; Jamieson, D. N.; Escott, C. C.; Hollenberg, L. C. L.; Clark, R. G.; Dzurak, A. S. Nature 2010, 467, 687-691.

(2) Maune, B. M.; Borselli, M. G.; Huang, B.; Ladd, T. D.; Deelman, P. W.; Holabird, K. S.; Kiselev, A. A.; Alvarado - Rodriguez, I.; Ross, R. S.; Schmitz, A. E.; Sokolich, M.; Watson, C. A.; Gyure, M. F.; Hunter, A. T. Nature 2012, 481, 344-347.

(3) Büch, H.; Mahapatra, S.; Rahman, R.; Morello, A.; Simmons, M. Y. Nat. Commun. 2013, 4, 2017.

(4) Simmons, C. B.; Prance, J. R.; Van Bael, B. J.; Koh, T. S.; Shi, Z.; Savage, D. E.; Lagally, M. G.; Joynt, R.; Friesen, M.; Coppersmith, S. N.; Eriksson, M. A. Phys. Rev. Lett. 2011, 106, 156804.

(5) Zwanenburg, F. A.; Dzurak, A. S.; Morello, A.; Simmons, M. Y.; Hollenberg, L. C. L.; Klimeck, G.; Rogge, S.; Coppersmith, S. N.; Eriksson, M. A. Rev. Mod. Phys. 2013, 85, 961.

(6) Muhonen, J. T.; Dehollain, J. P.; Laucht, A.; Hudson, F. E.; Kalra, R.; Sekiguchi, T.; Itoh, K. M.; Jamieson, D. N.; McCallum, J. C.; Dzurak, A. S.; Morello, A. Nat. Nanotechnol. 2014, 9, 986-991.

(7) Li, R.; Hudson, F. E.; Dzurak, A. S.; Hamilton, A. R. Nano Lett. 2015, 15, 7314.

(8) Maurand, R.; Jehl, X.; Kotekar Patil, D.; Corna, A.; Bohuslavskyi, H.; Laviéville, R.; Hutin, L.; Barraud, S.; Vinet, M.; Sanquer, M.; De Franceschi, S. arXiv:1605.07599. arXiv.org e-Print archive. http://arxiv. org/abs/1605.07599 (accessed May 2016).

(9) Kloeffel, C.; Trif, M.; Loss, D. Phys. Rev. B: Condens. Matter Mater. Phys. 2011, 84, 195314.

(10) Hao, X.-J.; Tu, T.; Cao, G.; Zhou, C.; Li, H.-O.; Guo, G.-C.; Fung, W. Y.; Ji, Z.; Guo, G.-P.; Lu, W. Nano Lett. 2010, 10, 2956.

(11) Higginbotham, A. P.; Kuemmeth, F.; Larsen, T. W.; Fitzpatrick, M.; Yao, J.; Yan, H.; Lieber, C. M.; Marcus, C. M. Phys. Rev. Lett. 2014, $112,216806$.

(12) Kloeffel, C.; Trif, M.; Stano, P.; Loss, D. Phys. Rev. B: Condens. Matter Mater. Phys. 2013, 88, 241405.

(13) Lauhon, L. J.; Gudiksen, M. S.; Wang, D.; Lieber, C. M. Nature 2002, 420, 57-61.

(14) Roddaro, S.; Fuhrer, A.; Brusheim, P.; Fasth, C.; Xu, H. Q.; Samuelson, L.; Xiang, J.; Lieber, C. M. Phys. Rev. Lett. 2008, 101, 186802. 
(15) Lu, W.; Xiang, J.; Timko, B. P.; Wu, Y.; Lieber, C. M. Proc. Natl. Acad. Sci. U. S. A. 2005, 102, 10046-10051.

(16) Hu, Y. J.; Churchill, H. O. H.; Reilly, D. J.; Xiang, J.; Lieber, C. M.; Marcus, C. M. Nat. Nanotechnol. 2007, 2, 622-625.

(17) Sercel, P. C.; Vahala, K. J. Phys. Rev. B: Condens. Matter Mater. Phys. 1990, 42, 3690.

(18) Csontos, D.; Brusheim, P.; Zülicke, U.; Xu, H. Q. Phys. Rev. B: Condens. Matter Mater. Phys. 2009, 79, 155323.

(19) Brauns, M.; Ridderbos, J.; Li, A.; Bakkers, E. P. A. M.; Zwanenburg, F. A. Phys. Rev. B: Condens. Matter Mater. Phys. 2016, 93, 121408.

(20) Fischer, J.; Coish, W. A.; Bulaev, D. V.; Loss, D. Phys. Rev. B: Condens. Matter Mater. Phys. 2008, 78, 155329.

(21) Hu, Y. J.; Kuemmeth, F.; Lieber, C. M.; Marcus, C. M. Nat. Nanotechnol. 2012, 7, 47-50.

(22) Higginbotham, A. P.; Larsen, T. W.; Yao, J.; Yan, H.; Lieber, C. M.; Marcus, C. M.; Kuemmeth, F. Nano Lett. 2014, 14, 3582-3586.

(23) Stangl, J.; Holý, V.; Bauer, G. Rev. Mod. Phys. 2004, 76, 725.

(24) Katsaros, G.; Spathis, P.; Stoffel, M.; Fournel, F.; Mongillo, M.; Bouchiat, V.; Lefloch, F.; Rastelli, A.; Schmidt, O. G.; De Franceschi, S. Nat. Nanotechnol. 2010, 5, 458-464.

(25) Ares, N.; Golovach, V. N.; Katsaros, G.; Stoffel, M.; Fournel, F.; Glazman, L. I.; Schmidt, O. G.; De Franceschi, S. Phys. Rev. Lett. 2013, 110,046602 .

(26) Ares, N.; Katsaros, G.; Golovach, V. N.; Zhang, J. J.; Prager, A.; Glazman, L. I.; Schmidt, O. G.; De Franceschi, S. Appl. Phys. Lett. 2013, 103, 263113.

(27) Koppens, F.; Buizert, C.; Tielrooij, K.; Vink, I.; Nowack, K.; Meunier, T.; Kouwenhoven, L.; Vandersypen, L. Nature 2006, 442, $766-771$.

(28) Mo, Y.-W.; Savage, D. E.; Swartzentruber, B. S.; Lagally, M. G. Phys. Rev. Lett. 1990, 65, 1020.

(29) Zhang, J. J.; Katsaros, G.; Montalenti, F.; Scopece, D.; Rezaev, R. O.; Mickel, C.; Rellinghaus, B.; Miglio, L.; De Franceschi, S.; Rastelli, A.; Schmidt, O. G. Phys. Rev. Lett. 2012, 109, 085502.

(30) Watzinger, H.; Glaser, M.; Zhang, J. J.; Daruka, I.; Schäffler, F. APL Mater. 2014, 2, 076102.

(31) Reilly, D. J.; Marcus, C. M.; Hanson, M. P.; Gossard, A. C. Appl. Phys. Lett. 2007, 91, 162101.

(32) Davies, J. H. The Physics of Low Dimensional Semiconductors; Cambridge University Press: Cambridge, U.K., 1998; pp 64, 98, 381.

(33) Haendel, K.-M.; Winkler, R.; Denker, U.; Schmidt, O. G.; Haug, R. J. Phys. Rev. Lett. 2006, 96, 086403.

(34) Nenashev, A. V.; Dvurechenskii, A. V.; Zinovieva, A. F. Phys. Rev.

B: Condens. Matter Mater. Phys. 2003, 67, 205301.

(35) Luttinger, J. M. Phys. Rev. 1956, 102, 1030.

(36) Bir, G. L.; Pikus, G. E. Symmetry and Strain-Induced Effects in Semiconductors; Wiley, New York, 1974.

(37) Lawaetz, P. Phys. Rev. B 1971, 4, 3460.

(38) Winkler, R. Spin-Orbit Coupling Effects in Two-Dimensional Electron and Hole Systems; Springer, Berlin, Germany, 2003.

(39) Van Kesteren, H. W.; Cosman, E. C.; Van der Poel, W. A. J. A.; Foxon, C. T. Phys. Rev. B: Condens. Matter Mater. Phys. 1990, 41, 5283.

(40) Winkler, R.; Papadakis, S. J.; De Poortere, E. P.; Shayegan, M. Phys. Rev. Lett. 2000, 85, 4574.

(41) Van Bree, J.; Silov, A. Y.; Van Maasakkers, M. L.; Pryor, C. E.; Flatté, M. E.; Koenraad, P. M. Phys. Rev. B: Condens. Matter Mater. Phys. 2016, 93, 035311.

(42) Zielke, R.; Maier, F.; Loss, D. Phys. Rev. B: Condens. Matter Mater. Phys. 2014, 89, 115438. 